\title{
Lapurdum
}

Euskal ikerketen aldizkaria | Revue d'études basques |

Revista de estudios vascos | Basque studies review

$17 \mid 2013$

Numéro XVII

\section{Topónimos alaveses de base antroponímica terminados en -iz, -ez y -ona / -oa}

Patxi Salaberri Zaratiegi

\section{OpenEdition}

\section{Journals}

Edición electrónica

URL: https://journals.openedition.org/lapurdum/2445

DOI: 10.4000/lapurdum.2445

ISSN: 1965-0655

\section{Editor}

IKER

Edición impresa

Fecha de publicación: 1 octubre 2013

Paginación: 201-220

ISBN: 978-2-86781-409-9

ISSN: $1273-3830$

Referencia electrónica

Patxi Salaberri Zaratiegi, «Topónimos alaveses de base antroponímica terminados en -iz, -ez y -ona / -oa», Lapurdum [En línea], 17 | 2013, Publicado el 15 noviembre 2015, consultado el 22 julio 2021. URL: http://journals.openedition.org/lapurdum/2445 ; DOI: https://doi.org/10.4000/lapurdum.2445 


\section{Topónimos alaveses de base antroponímica terminados en -iz, -ez y -ona /-oa}

Patxi SALABERRI ZARATIEGI

UPNA / NUP

\section{Laburpena}

Giza izen bat oinarri eta -iz, -ez eta -ona / -oa amaierak dituzten Arabako leku izenak

Lan honetan oinarritzat latin jatorrikoa ohi den jende izena eta atzizkitzat -iz (euskara eta gaztelania), -ez (gaztelania) eta -ona (gaztelania), -oa (euskara) duten Arabako toponimoak aztertzen ditugu. Zenbait ikertzailek atzizkien etorkiaz jaulki ustekariak aipatu ondoren, -iz, -ez-en iturritako -(o)nis genitiboa proposatzen dugu, -icus atzizkiaren -ici genitiboa ezin bazter badaiteke ere. Garbi dago une batetik aitzina jabegoa adierazten zuen -(i) (t)z atzizkia beregaindu zela, bertako oinarriei atxiki zitzaiela, eta, beraz, ez dela nahitaezkoa beti latinera jotzea. Bestalde, -ona, -oa berrekailuari doakionez, beste autore batzuek errandakoari jarraikiz, $-o$, -onis ereduaren arabera deklinatu izenen -one ( $m$ ) akusatiboa proposatzen dugu iturburutzat, gero -ona, -onae deklinabidera egokitu zena.

Giltza hitzak: Arabako toponimia, deantroponimikoa, antroponimoa, atzizkia, bilakaera fonetikoa

\section{Abstract}

Placenames of Álava with anthroponymical bases and ending in -iz, -ez and -ona / -oa

In this work we analyze the alavese toponyms that are based on a person name, whose origin is usually Latin, in addition to these toponyms having suffixes like -iz (Basque and Spanish), -ez (Spanish) on the one hand, and -ona (Spanish), -oa (Basque) on the other. After dealing with what various authors have claimed about the origin of said suffixes, we propose the Latin genitive ending -(o)nis for $-e z$, -iz, albeit the genitive form -ici of the suffix -icus can not be discarded here either. It is clear, in any case, that from some point on the $-(i)(t) z$ suffix 
that denoted ownership became an independent suffix on its own right, being subsequently applied to native names, which shows us that it is not always necessary to resort to Latin. On the other hand, regarding the suffix -ona, -oa, which also denotes ownership, we propose, following what other authors have claimed about its origins, the accusative ending -one $(m)$ as the original form, which appeared first in names declined following the pattern -o, -onis and eventually became adapted to the declension -ona, -onae. evolution

Keywords: Alavese toponymy, deanthroponymic, anthroponym, suffix, phonetic

\section{Topónimos en -iz, -ez}

Mitxelena, en su conocida obra Apellidos Vascos (347), menciona los apellidos y topónimos Ajanguiz, Betriquiz, Berriz, Eneritz, Gauteguiz, Mandaluniz, Ocariz, Olondriz, Oloriz, Sangroniz, etc., y considera que en el final que todos muestran se reúnen formaciones muy diversas por su origen. Cree que en nombres como Othaitz (Oteyza), Olaiz (Olaizola), etc. podemos estar ante el sufijo -itz «fundamentalmente igual a otros sufijos en tz que expresan pluralidad» que menciona Lafon (1980 [1944]: 530), y añade que en opinión de Garate (1931: 446-447) es idéntico a -tza. Entre los topónimos que este último autor menciona están los siguientes: Alaiz / Alaiza, Araiz / Araitza, Ariz / Aretxa, Arraiz / Arraitza, Artaiz / Artaza, Barkoiz / Barcus / Parkotxa, Beraiz / Beraza, Heriz / Erize, Olaiz / Olatza y otros. Estima Mitxelena (AV, 347) que en la lista de Garate «se reunen cosas muy heterogéneas: son numerosos los ejemplos no de -iz / -za, sino de -iz / -iza», pero cree que en el caso de Othaitz y Çeapeiz (topónimo medieval de Pamplona formado sobre zeape, variante de ziape 'mostaza') la opinión de dicho autor es valida.

Añade Mitxelena que en opinión de S. Arana (1980 [1897]: 710-712, 739-741) el final -iz es el mismo que encontramos en los patronímicos españoles del tipo Sánchez, Sánchiz, Sanchis, y que Caro Baroja (1945: 102-106), en la misma línea, sostuvo que un número abundante de nombres de poblaciones en -iz proceden del genitivo -ici de nombres en -icus formados sobre antropónimos: Albeniz (Albinus), Apellaniz (Ampellius), Apraiz (Aper, Apraicus), Belendiz (Plend[i]), Gordeliz (Gordelius), Trocóniz (Troconius), etc.

Apunta el lingüista guipuzcoano (ibíd.) que «parece haberse empleado bastante, en tiempos relativamente recientes, para formar derivados de otros topónimos o apellidos: Aldama / Aldamiz, Arrona / Arroniz, Echano / Echaniz, Garro / Garriz, Garroiz; Guereca / Guerequiz, Lemona /Lemoniz, Lezama / Lezamiz, Marquina / Marquínez, Munita / Munitiz, Orba / Orbaiz, Orbaiceta». Como resumen considera que «la mayoría de los nombres aquí mencionados no tienen, hasta ahora, explicación vasca; tampoco la tienen a fortiori, latino-románica». Según Menéndez Pidal y Tovar (1962: 446) las formas derivadas en -iz tienen, respecto a las primitivas, «un sentido adjetival de pertenencia o correlación» y es posible que así sea, pero nosotros no lo vemos del todo claro, en el caso de los topónimos vascos como Etxaniz, Lemoiz (< Lemoniz), Munitiz frente a Etxano, Lemoa (< Lemona), Munita.

Creemos que, en general, aunque hay excepciones, los topónimos en -iz (euskera y castellano) / -ez (castellano) son formaciones basadas en nombres de personas e, igualmente, que el final -iz / -ez es el mismo que encontramos en las formaciones patronímicas (véase Salaberri, 2003), es decir, en aquellas como Garzea Enekoitz, Dota Azeariz en las que se 
indicaba que el portador o portadora del que vamos a denominar "identificador" (el elemento que se corresponde con el moderno apellido) en -(i)(t)z era hijo o hija de Garzea y Azeari respectivamente. De la misma manera, Ilurdo(i) (t)z podía ser el hijo o hija de un tal Ilurdo, Ilurde, pero también la posesión de la persona así llamada. Por eso encontramos Ilurdotz como patronímico e igualmente como nombre de una localidad del valle de Esteribar cercana a Pamplona / Iruñea. La variación -i(t)z / - $(t) z$ es típica de los patronímicos (cf. Enekoi $(t) z$, Eneko $(t) z$, Eneki $(t) z$, etc.), y está presente también en los nombres de lugar, aunque estos se fijaron, habitualmente (no siempre), de una u otra manera en fecha temprana (compárese Agoitz con Oskotz, ambos en Navarra).

En lo que respecta al origen del sufijo, considero, siguiendo a Mitxelena (1957) e Irigoien (1987) que estamos ante un descendiente del genitivo latino que indicaba posesión, en el caso de los patronímicos 'hijo de', 'hija de' y en el de los topónimos 'posesión de', 'terreno de', 'caserío de'. Creo que las formas en -oitz proceden de formaciones del tipo Enneconis 'de Eneko', 'de Íñigo', es decir, de formas conjugadas por la tercera declinación latina en -o, -onis, muy frecuentes en la Edad Media, aunque es posible también que el sufijo en -ici tuviera que ver en la creación de los patronímicos, y, en consecuencia, también de los topónimos, ya que, como se ha dicho, la formación es la misma en ambos casos, si bien cada uno de ellos tiene sus especificidades.

No obstante, lo anterior no quiere decir que tengamos que recurrir al latín para dar cuenta de todos y cada uno de los patronímicos y topónimos. Patronímicos del tipo de Ilurdo(i) tz, Otsando(i)z, Otsoko(i) (t)z, Zuritz, etc., demuestran claramente que el sufijo era independiente, tenía varios alomorfos -que dependían en cierta medida de la forma del nombre, que no era la misma en todos los casos- y se podía añadir a bases indígenas que no tenían nada que ver con el latín. Que los patronímicos indicaban un cierto tipo de pertenencia es claro ('hijo de', 'hija de') y también que los topónimos similares o idénticos a ellos expresaban en principio posesión, característica que se muestra de manera nítida en topónimos (y patronímicos) que contrastan con otras formaciones que llevan otro sufijo de pertenencia: Beraskoitz (actual Beskoitze en Lapurdi) vs. Beraskoain (N, variante eusquérica de la forma castellana Belascoáin, sobre Berasko / Velasco, de origen germánico), Garzei(t)z vs. Gartzain (N, ambos construidos sobre Gar(t)ze), Otsokoiz vs. Otsokain > Otsakain (N, con base Otsoko, hipocorístico de Otso(a)), Barañain (N) vs. Barañao (B, Barañano en castellano, ambos tal vez de Veranius, si bien no se puede descartar que el antropónimo de base sea Varanius), Zuritz (pat.) vs. Zuriain (N, con base en el antropónimo Zuri; había también Zurikoain en Navarra, convertido ahora en Zurukuain), etc.

El problema que nos surge en el caso de los topónimos con final en sibilante es saber en qué casos el topónimo procede directamente de una forma latina, y en cuáles podemos o debemos pensar en una formación posterior, medieval. Este problema está presente también en el caso de otros sufijos, como por ejemplo en el de -ain que muestran los ya mencionados Gartzain, Otsokain, Zuriain, Zurikoain, y el no mencionado Andrekiain, formado sobre un hipocorístico Andreki con base en Andre(a) (cf. Andre 'señora').

Sería muy interesante reflejar en un mapa de Vasconia la distribución geográfica de los diferentes sufijos que están presentes en nuestros topónimos, pero antes hemos de identificar todos los que existen y, para ello, es necesario dar una explicación etimológica satisfactoria de muchos de nuestros nombres de lugar. Por ejemplo, y sin salir de la parte occidental de la zona de habla vasca, tenemos el sufijo - (i) ano en Bilbao / Bilbo, -inu en Andollu, -itu en Jungitu, etc. 
Otro punto interesante, en el tema que nos afecta aquí, es la relación de los nombres en -i $(t) z$ y los en $-o(t) z$, estos últimos presentes en la parte centro-oriental de Vasconia. En otro trabajo (Salaberri, 2011b) hemos derivado algunos de los topónimos en -o(t)z, -oze -que tradicionalmente se explicaban como basados en el sufijo pirenaico homónimo- del genitivo latino en -onis ya mencionado, es decir, de formaciones declinadas como -o, -onis que se vieron reducidas, al igual que muchos de los patronímicos, a -otz. Sin embargo, decíamos en aquel trabajo que el sufijo en realidad no es -otz sino - tz, ya que la vocal -o es parte del antropónimo de base, no del sufijo (Enekoitz<Enneco/nis). Cuando el final -i $(t) z$ se hizo idenpendiente y se sintió como de pertenencia, se empleó para crear patronímicos del tipo de Zuritz y topónimos como Gasteiz, y a eso se debe la gran cantidad de topónimos con final en -(i)tz, entre los que se cuentan también los en -o(t)z, en los que, como hemos señalado, la vocal es parte del tema, no del sufijo. Estos últimos (los en -o $(t) z$ ) tienen a veces variantes antiguas en -oi $(t) z$ que indican claramente cuál es la procedencia del sufijo (Açoiz / Azotz, N), si bien la mayoría de ellos no muestran dicha alternancia. En alguna ocasión (en Agoitz, N, por ejemplo), tenemos siempre la variante acabada en -oitz, con -o que pertenece a la base.

Antes de pasar a examinar uno por uno los nombres de localidades alavesas acabados en sibilante, es preciso señalar que no tenemos ningún testimonio de que dicho sonido haya sido africado en algún momento de la historia en la mencionada región. Es un tema delicado, pero los datos que tenemos hacen pensar que el final era un sonido fricativo dorsoalveolar, presente también, en origen, en numerosos nombres de localidades vizcaínas, si bien con posterioridad, en estos últimos, dicho sonido se neutralizó a favor de la apico-alveolar correspondiente.

Examinamos a continuación, por orden alfabético, los topónimos alaveses en -iz, -ez:

\section{Albeiz / Albéniz (Asparrena)}

En 1025 (Ubieto, 1976, 180, p. 177) hay Albiniz y en 1062 (Lacarra, 1965, 23, p. 32) Senior Scemeno Consaluoç de Alueniç. En 1232 en Irantzu (Jimeno, 1970: 248) tenemos las ruedas d'Albiniz al lado de las ruedas de Haraya; en 1257 se documenta Aluiniz (Rodríguez, 1989, 235, p. 217), hacia 1275 don Garçia d'Alueniz (ibíd., 340, p. 304), en 1351 Alvenis (Iñurrieta, 1989, 61, p. 95), en 1471 «Pero Martines de Aluenis, alcalde de la Hermandad de Heguilas» (Ayerbe, 2006, 244, p. 139), en 1484 «Sennor Joan Peres de Aluenis alcalde de la Hermandad de la villa de Salvuatierra de Alaba e su juridiçion» (ibíd., 257, p. 185). En microtoponimia vasca hay Albeiz(Albeizbidea, Albeizmendiara, Albeizmendiostea, 1625).

Gómez-Moreno (1925: 491) destaca el parecido existente entre Albéniz y el nombre Albennes de la onomástica ibérica. Caro Baroja (1945: 103) cree que Albéniz ha salido de Albanici y este, a su vez, del antropónimo Albanus o Albinus al que se le ha añadido el sufijo -icus en genitivo, presente también en los patronímicos como López, de Lupici, «genitivo singular y nominativo plural» de Lupicus. Menéndez Pidal y Tovar (1962: 390) consideran que Albéniz, documentado Albiniz en 1025, procede de «Albenus, que con sufijo-acu- da Aubenac (Dordogne), o de Albinus». Independientemente del origen del sufijo, yo también creo que en la base hay un antropónimo, Albinus en mi opinión. La evolución habrá sido la siguiente: Albiniz (1025) > Albeniz (por disimilación de vocales) > Albéniz / Albeiz (por caída de la nasal lene en posición intervocálica).

Mitxelena $(A V, 30)$ en la entrada dedicada a Albi- dice que «parece haber sido un 
nombre personal a juzgar por el ap. Albirena», y apostilla que Albiz parece un patronímico. En la entrada siguiente explica que esta última forma «puede muy bien ser una reducción de Albeniz, Albiniz, por pérdida de la -n-». En otro trabajo (1956: 341-342) escribe el lingüista guipuzcoano que «la comparación de nombres de poblaciones en -iz e -iniz hace pensar que podemos estar ante variantes del mismo nombre, unas más conservadoras y otras que reflejan la evolución popular», y cita como ejemplo Albiz (barrio de Mendata, B) frente a Albeniz (Albiniz) (A), Gamiz (A) frente a Gaminiz (B), y Ariz barrio de Basauri (B) y pueblo de Navarra, frente a Ariniz (A, 1025) «hoy Arínez».

\section{Apelaiz / Apellániz (Arraia-Maeztu)}

En la Reja de San Millán, en 1025, se documenta como Apinganiz, es decir, Apiñaniz, con nasal palatal, y en la actualidad, según López de Guereñu (1989: 615), se sigue diciendo Apiñaniz, a pesar de que la variante disimilada Apillaniz, con lateral palatal, aparece por primera vez en 1257 y el nombre oficial de la localidad ha venido siendo Apellániz.

En 1110 tenemos Apignaniz (Martín Duque, 1983, 239, p. 326), en 1257 Apillaniz (Rodríguez, 1989, 235, p. 236) y en 1299, en Estella, se documenta «Garcia Periz fill de don Pero Garçez de Apinaniz, quarpenters» (García Larragueta, 1976-77, 105, p. 545). En 1456 un vecino de Cicujano, aparece indistintamente como Juan de Apeñaniz y Juan de Apellaniz (Pozuelo, 1998, 6, pp. 23-24). Martínez de Madina (2010: 310) recoge la forma vasca Apelaiz, sin ningún añadido, en 1531, 1633 y 1635, forma que alterna, en la documentación escrita integramente en idioma distinto al euskera, con la versión romance: Apellaniz (1536), Apilaniz (1546, 1557, 1586, 1637), Apelaniz (1577, 1584, 1593, 1598, 1630), Apelanis (1588), etc.

En microtoponimia vasca encontramos a menudo alguna variante de la forma romance, cosa nada extraña (Apelaniz gana, 1593; Apelanizgain, 1602; Apelanizgana, 1630; Apellanizgana, 1633, 1710 y 1763; Apilanizgana, 1701, etc.; Apelaniz ostea, 1687; Apilaniz ostea, 1687; Apellaniz ostea, 1688; Apilanizostea, 1688; Apillanizostea, 1688, etc.; Apelanizbea, 1594; Apellaniz Vea, 1657; Apelanizbea, 1711; Apelanispea, 1727), pero los topónimos que recogen el nombre eusquérico de la localidad, es decir, la forma que muestra la caída de la nasal lene en posición intervocálica, son muy numerosos: Apilaizbeaque (sic, 1557), Apilayzpea (1570), Apellaezpea (1592), Apilezbea (1594), Apilezgana (1610, 1626), Apilazbea (1616), Apillaizgana (1637), Apelaisgana (1641), Apellaizgana (1658), Apilasosttea, Apilas osttea (1691), Apelaiz ostea (1693), Apilazostea (1703), etc. (Martínez de Madina, 2010: 310-313; véase también López de Guereñu, 1989: 46).

Caro Baroja (1945: 103) propone Ampelici como base y Menéndez Pidal y Tovar (1962: 390) ven Apinianus o Pinianus en la raíz del topónimo, pero parece más conveniente pensar en Appinius, al que se le habría añadido en un principio el sufijo -anu, o sea, habrá que partir de *(fundu) appinianu > *Apiñano, forma que habría tomado, ya en la Edad Media, el sufijo -iz (cf. Etxano / Etxaniz y, sobre todo, Markiz / Marquinez, Marquina de iuso y Marquina de suso en 1025), convirtiéndose en Apiñaniz.

De Apiñaniz, por disimilación de palatales (Mitxelena, 1972: 22), ha salido Apillaniz (aunque, como hemos visto, Apiñaniz está todavía vivo), forma que, en castellano, por disimilación de vocales o apertura de la vocal cerrada en contacto con una consonante palatal (Apillaniz > Apellaniz; vid. Mitxelena, ibíd., 23), se convirtió en Apellániz, que conserva la vocal final cerrada. En esto no hay un único resultado (cf. Gamíniz / Gamiz en B, o Albéniz / Albeiz 
frente a Ariñez / Ariz, Marquínez / Markiz sin salir de Álava).

En euskera, del original Apillaniz saldría Apillaiz y con despalatalización propia de ciertas hablas alavesas, Apilaiz y, en composición, Apilaz-, Apilez-, la primera con reducción del diptongo -ai- >-a- y la segunda con monoptongación -ai->-e-. La variante Apellaiz- habrá salido, tal vez, de Apellaniz, con pérdida de la nasal; Apellaez- se deberá a asimilación.

\section{Arangiz (Gasteiz)}

Arangiz (1025, Reja de San Millán), «Senior Xemeno Gundessalboiz de Aranquiz, fide» (1066, Martín Duque, 1983, 79, p. 122), «De monasterii Sancti Vincenti de Ocoizta Et ego senior Alvaro Gonzalvez de Aranguiz vendi tibi dompno Nunno, abbati de Ocoizta, de mea erentia quam habui in Ocoizta [...]» (1084, Ledesma, 1989, 91, p. 70), Aranguiz Gorostiçaha (1295, Rodríguez, 1989, 518, p. 447), Aranguis (1409, Ruiz de Lóizaga, 1997, 19, p. 94).

Mitxelena $(A V, 56)$ no ve claro si es un derivado de angio 'dehesa, lugar de pasto acotado' o no. Nosotros, por nuestra parte, no podemos asegurar que se trate de un deantroponímico, aunque tampoco podemos descartar que lo fuera. De serlo, habría que pensar en un nombre personal *Alanc(i)us, *Aranc(i)us o similar que no encontramos, o tal vez en *Calanc(i)us, ${ }^{*}$ Caranc(i)us.

\section{Argomaiz / Argómaniz}

En la Reja de San Millán, en 1025, hay Arguimaniz según Ubieto y Argumaniz según otros editores. En 1256 (Jimeno, 1970: 247) y en 1295 (Rodríguez, 1989, 518, p. 448) tenemos Argumaniz, por lo que parece que habrá que pensar en una variante de *Argumana provista de -iz (cf. Marquina de suso, Marquina de iuso / Marquínez / Markiz). No obstante, en 1257 hay Argomaniz, con o (Rodríguez, 1989, 235, p. 218) y también en 1295 (Rodríguez, 1989, 512, p. 438) y 1510: «... en el lugar de Argomaniz termino llamado Guevararavidea [...] teniente al camino que van de Argomaniz a Guevara» (1510, Lema, 2007, 59, p. 108).

El origen podría ser *(villa) argumana, basada en un antropónimo *Argumus que no hemos encontrado. La apertura $u>o$ habría que achacársela al romance, pero, de todas las maneras, en microtoponimia eusquérica hay Argomaiz- (Argomaizpide, Arbulu, 1799, López de Guereñu, 1989: 61), además del tardío y (por ello) reducido Argomas- (Argomaspide, Matauku, 1865) y el castellano Argomaniz- (Argomanizbidea en Oreitia en 1696, Argomanizbide en Arbulu en 1760 y en Matauku en 1797). Es decir, hay formas con -o- bastante tardías. Es conocida también la tendencia presente en algunos casos a convertir una vocal en $u$ ante $m$ (decima $>$ dekuma).

\section{Ariz / Aríñez (Gasteiz)}

Ariniz (Reja, 1025), Ariniz (1173, Rodríguez, 1979, 2514, p. 30), Arriniz (1257, Rodríguez, 1989, 235, p. 228), Harenis (1295, ibíd., 512, p. 439).

Caro Baroja (1945: 103) clasifica Arínez con Albéniz, Apéllaniz y otros topónimos de origen antroponímico con final procedente de -ici, pero no dice cuál es el antropónimo de base. Explica, eso sí, que en la documentación de San Millán hay Albiniz, Ariniz, etc., «nombres (que) han sufrido la transformación normal en castellano de la "i" en "e"» (ibíd., 106). 
Como se ha dicho (vid. Albeiz / Albéniz), Mitxelena creía que, vistos los finales -ize -iniz con la misma base, podríamos estar ante variantes de un único nombre, unas más populares (las en -iz) y otras más conservadoras (las en -iniz): Albiz / Albeniz (Albiniz), Gamiz / Gaminiz (B), Ariz barrio de Basauri (B) y pueblo de Navarra frente a Ariniz (A, 1025), «hoy Arínez». No obstante, dice el lingüista de Errenteria $(A V, 80)$ que Arínez podría ser un derivado de $\operatorname{ar}(h)$ in 'ligero, ágil', cosa en nuestra opinión sumamente rara en este tipo de topónimos.

Creemos que se trata, como también seguramente en el caso de Aria barrio de Aberasturi (A), de un derivado *Arina formado sobre el antropónimo Arius y dotado del sufijo -ina, al que, con posterioridad, se le ha añadido -iz / -ez, como en Aldama / Aldamiz, Etxano / Etxaniz $(A V, 347)$. No es posible considerarlo un derivado de Arina, despoblado de Ganboa, ya que este exige un étimo con nasal fortis (Alinna), razón por la cual la nasal no cae en posición intervocálica en la variante vasca. Según la Comisión de Onomástica de Euskaltzaindia (en prensa) la forma eusquérica del topónimo es Ariñiz, presente en microtopónimos como Ariñizbidea, de la que Ariñez habrá salido por acomodación a los usos de la fonética castellana, como Arkauti > Arcaute, Artzi > Arce, Oñati > Oñate, etc.

Sin embargo, nosotros pensamos que Ariniz, documentado todavía en 1714, dio lugar a Ariz, que es la forma que se documenta en el topónimo Arizbidea, Arizpidegana 'el camino de Aríñez', 'lo de encima del camino de Aríñez' de la vecina localidad de Lermanda. La evolución romance habrá sido Ariniz > Aríniz (con palatalización debida al entorno palatal) > Arínez (con apertura de la vocal final habitual en los topónimos romances; cf. Muskiz / Musques en Bizkaia o Dordóniz / Dordonez [1416] y Markiz / Marquinez en la propia Álava), y la eusquérica, por su parte, Ariniz > *Arìiz > Ariz.

\section{Astúlez (Gaubea / Valdegobía, A)}

Astulez (1290, López de Guereñu, 1989: 620; cita de Landázuri), «... la villa de Salinas de Añana e Caraca e Astulez e Sobron e la Puente de Larra» (1460, Pozuelo, 2008, 28, p. 59), «... las alcaualas y salinas de la vuestra villa de Salinas de Anaya (sic) y Atiega su aldea [...] los lugares solariegos Caranca e Astulez e Sobron [...] e Retuerto e Marquina» (1481, ibíd., 42, p. 94). Parece tratarse de un derivado en -ez de un nombre *Astul, quizás variante del documentado Astur, Asturius, Asturus.

\section{Dordóniz (Trebiñu)}

Sancti Cipriani de Dordoniz (1110, Martín Duque, 1983, 239, p. 326), «Dono etiam Sanctum Ciprianum de Dordoniz» (1113, ibíd., 254, p. 343), Dordoniz (1257, Rodríguez, 1989, 235, p. 222), Dordonis (1415, Ruiz de Lóizaga, 1997, 29, pp. 107, 108, 109), Dordonez (1416, ibíd., 31, 110).

Parece que la variante vasca tendría que ser *Dordoiz, que no hemos documentado. En cuanto a la etimología del topónimo, queremos señalar, como mera posibilidad, que tal vez se trate de un derivado del nombre personal (medieval) Dordonius, con el sufijo -iz. Se documenta otro Dordoniz «in Archipresbyteratu de Rio de Oia» en 1257 (Rodríguez, 1989, 235, p. 230). 


\section{Estibaliz (Gasteiz)}

«Regnante Sancio rex in Pampilona, comite Lupe Sarrazinez in Divina, Aurivita Didacoz in Estivaliz» (984, Ubieto, 1976, 98, p. 113), «In Alaua Sanctam Mariam de Estiualiz cum ipsa uilla et omnibus suis pertinenciis» (1175, Rodríguez, 1979, 252, p. 38), «... qui migrauerunt de Uilla Franca de Stiualiz ad Uictoriam» (1193, ibíd., 335, p. 120), «... ecclesiam sive cappellam beatae Mariae Grangiae loci de Eztibalis» (1417, Ruiz de Lóizaga, 1997, 34, p. 117).

Mitxelena $(A V, 347)$ estima que la idea de Gorostiaga según la cual Estivaliz procede del latín vallis tropieza con la dificultad que supone el hecho de que la forma vasca sea Estivariz, por lo que no sería posible que - $r$ - procediera de -ll-. Considera preferible la etimología *Aestivalis que el mismo Gorostiaga propone y rechaza. Estibalis (una vez Eztibaliz), Estibariz, Estibaris es un nombre personal, la inmensa mayoría de las veces masculino, muy habitual en la Edad Media, pero no parece, al contrario de lo que opina Del Valle Lersundi (1933: 178), que se deba a la devoción a la virgen alavesa de Estibaliz.

En lo que concierne a la etimología del nombre, si partimos de la forma *Aestivalis que propone Mitxelena (Aestivus está documentado), tendríamos que pensar que el final de la base se reinterpretó como si fuera el sufijo procedente del genitivo latino, dada la cercanía fónica y gráfica existente entre ambos, favorecida sin duda por variantes del sufijo patronímico en -is, presentes también en cierta medida, por otra parte, en los topónimos provistos de dicho final. Otra posibilidad sería partir del nombre personal *Estival y considerar que se le añadió el mismo sufijo que llevan los topónimos que estamos analizando en este trabajo.

\section{Gamiz (Gasteiz)}

Gamiz (1025, Reja; 1257, Rodríguez, 1989, 235, p. 219; 1295, ibíd., 512, 519, pp. 439 y 448; 1351, González, 1994, 8, p. 35), Iohanni de Gamis de Gamboa (1456, Ruiz de Lóizaga, 1997, 106, p. 188).

En al caso de la localidad alavesa podríamos partir del antropónimo Camius, es decir, derivarlo de *(fundus, ager) camici de donde Gamitz por sonorización de la velar sorda inicial y evolución del final asibilado -ici > -itz (e) > -itz, proceso ocurrido seguramente antes de su incorporación al euskera. Como se ha visto más arriba, Mitxelena (1956: 341-342) pensaba que en el caso de los topónimos parecidos pero con final diferente en -iz / -iniz como Albiz / Albiniz (> Albéniz / Albeiz), Ariz / Ariniz (> Arínez) o Gamiz / Gaminiz, la primera forma de cada par era la versión popular, mientras que la segunda correspondía a la culta.

No obstante, hay que tener en cuenta, a mi parecer, que Albiz puede ser el fruto de añadir - $(t) z$ directamente a Albi, ya que el sufijo, como se ha dicho al comienzo de este trabajo, se hizo independiente y se pudo emplear con cualquier base (cf. el patronímico Zuritz, sobre Zuri). Por lo tanto, no es necesario aceptar que se produjo la evolución Albiniz > Albiz, a pesar de que es posible que así haya ocurrido.

Para el Gaminiz vizcaíno, llamado en euskera Gamiz, podemos partir de Caminius, de * (fundus, villa) caminici, de donde Gamíniz en romance y Gamiz en euskera, con pérdida de la nasal lene en posición intervocálica y contracción de las vocales iguales: *Gáminitz > *Gámîitz > Gámiz. Sin embargo, también podemos unir el topónimo alavés y el vizcaíno, si aceptamos que ambos han salido de un derivado del mencionado Camius, es decir, de *Gámina (véase, a propósito de los topónimos en -inu / -ina, Salaberri, 2012b), de donde *Gáminitz > Gáminiz 
(con neutralización a favor de la fricativa, en época medieval probablemente) > *Gámîiz > Gámiz. Que en el caso de la localidad alavesa no haya ningún testimonio de la forma Gaminiz puede parecer extraño, pero téngase en cuenta que tampoco lo hay de *Zeriano en el caso de Zerio, a pesar de que otro topónimo del mismo origen, Ciriano (Zirao en euskera), todavía hoy en día conserva la forma cercana al étimo (véase Salaberri, 2011: 146-147 y 2013: 266).

\section{Gasteiz (Vitoria)}

El nombre eusquérico de la capital de Álava se documenta por primera vez como Gastehiz en 1025 en la Reja de San Millán (Mitxelena, 1964: 26, Ubieto, 1976, 180, p. 176, Caro Baroja, 1980: 112), y después como Gasteiz en 1089 (Alvaro Gonzalvez de Gasteiz, Ledesma, 1989, 186, p. 129) y en 1135 (Rodríguez, 1992, 108, p. 178), y como Gasteys en 1181 (Crespo et al., 1995, 1, p. 1). En 1193 tenemos "Petro Remiri [tenente], Victoriam» (Jimeno, 1968, 145, p. 249) y «ad Uictoriam» (Rodríguez, 1979, 335, p. 120), en 1198 Victoriam (1198, Goñi, 1997, 423, p. 366), en 1257 Bitoria (Rodríguez, 1989, 235, p. 220), en 1310-12 «apud Bitoriam», «... fuimus ad Bitoriam» (Ruiz de Lóizaga, 1997, 1, p. 66), «Item liber de Bitoria» (ibíd., p. 68), en 1387 hay de nuevo Bitoria (ibíd., 9, p. 81), en 1420 «in villa de Victoria» (ibíd., 43, p. 124), en 1421 «... in villa de Bitoria» (ibíd., 52, p. 131) y en 1425 «... conventus monasterii de Victoria» (ibíd., 65, p. 141).

Es en mi opinión un derivado del antropónimo Gaste, Gastea bien documentado y relacionado con el nombre Garze, Garzea, Garzia, conservado en el apellido García. Para más detalles pueden verse los trabajos de Irigoien (1982), Iglesias (2001, 2002) y Salaberri (2005: 612-615 y 2011c: 90-91).

\section{Gordeliz (barrio de Artziniega)}

Gordelliz (1798; López de Guereñu, 1989: 632, que lo toma de Landázuri). No poseemos ningún dato documental antiguo y, en estas circunstancias, es sumamente arriesgado intentar aclarar el origen del topónimo. Parece uno de los numerosos nombres de lugar en -iz que estamos examinando en este trabajo, es decir, un antropotopónimo, basado tal vez en una variante ${ }^{*}$ Cordellus del documentado Cordulus.

\section{Gurbandiz (despoblado de Trebiñu)}

Gurvandiz (1257, Rodríguez, 1989, 235, p. 222), Gurmandiz (término de San Vicentejo, 1762, 1773-1778, 1802; González de Viñaspre y Uribarrena, 2012: 434-435).

Según González de Viñaspre y Uribarrena (ibíd., 435) «... parece un compuesto con sufijo $-i(t) z$, frecuente en toponimia, que suele ir añadido a un nombre de persona. En este caso no se ha identificado el antropónimo». Este podría ser, en nuestra opinión, algo como *Curvantius, posible variante del documentado Curvatius, aunque no es fácil explicar el desarrollo de la nasal. Otra posibilidad, menos probable a nuestro juicio, es ver en la base una variante *Gurgantius de Gurgentius, de donde, por disimilación de velares, habría salido *Gurbantius. Es decir, necesitaríamos la base *Curvantici o *Gurbantici para llegar a Gurbandiz, si no se piensa que, como en el caso de Markiz / Marquínez, el sufijo se le ha añadido a una base en -ina derivada de los mencionados antropónimos, es decir, a *Curbantina, *Gurbantina. En 
este caso, sin embargo, no hay ningún rastro de ello.

\section{Langraiz Oka / Nanclares de la Oca (Iruña Oka)}

Langrares (hermandad, Reja, 1025), Lancrares (1257, Rodríguez, 1989, 235, p. 227), Lanclares (1295, Rodríguez, 1989, 512, p. 438).

No vemos del todo claro que se trate de un topónimo basado en un antropónimo. Parece que tendría que estar relacionado, etimológicamente, con Langara-Ganboa o Nanclares, cuyos testimonios documentales coinciden parcialmente con los del topónimo que estamos examinando: Langara et Moio (Reja, 1025, en Camboa), Sancho Langara (1247, García Larragueta, 1957,313 , p. 308), Lancrares Moyo (1257, Rodríguez, 1989, 235, p. 218), Domingo de Lancrares (1279, García Larragueta, 1957, 455, p. 485), Lanclares de Gamboa (1295, Rodríguez, 1989, 512, p. 438 e ibíd., 518, p. 448), Juan Saez de Nanclares (1480, Iñurrieta, 1983, 17, p. 40; este podría corresponder a cualquiera de los dos Nanclares actuales).

Mitxelena (1950: 478, AV, 317) relaciona Gabalaika, Guevara con el gótico gibla y el griego $\kappa \varepsilon \phi \alpha \lambda \eta$, y dice que parece documentarse aquí, por primera vez, el sufijo -ika presente en Langarika, relacionado con Langara, «que puede ser el mismo Láncara de León y Lugo», pero muestra la sonora típica tras sonante en lengua vasca.

Si se aceptara que hay un antropónimo en la base, tendríamos que ver algo como *Lanc(r)arus, *Lang(r)arus que podría proceder, igualmente, de una forma con grupo $\mathrm{cl}$-, fl-, pl- en euskera. En mi opinión es posible que de *(villa) lanc(r)arana, *(villa) lang(r)arana saliera *Langarana, de donde *Langarâa > Langara. Langarica, por su parte, habría salido de * (villa) lang(r)arica o * (villa) lanc(r)arica, con sufijo -ica en lugar de -ana. El problema que esta propuesta presenta es que se pierde la posible conexión con el Láncara mencionado de otras regiones. El final -ara, sea una coincidencia o no, lo encontramos en Aistara y en Bokara, sin salir de Álava.

García Arias (2005: 121-122), al hablar del topónimo asturiano Llangreu dice lo siguiente:

«Para algunos, el nombre del concejo centroasturiano tendría un origen indoeuropeo. Para ello sospechan que se formaría sobre una palabra *LANKA 'hundimiento', 'hondonada' que habría de verse en el posible celtismo *LANKA interpretado como 'lecho de un río', quizá extensible a 'valle', y en el inicio de una formación *LANKARA $\rightarrow$ *LANKAREU».

Sin embargo, él prefiere derivar el topónimo del latín plancam 'madera, plancha de madera' que en asturiano pervive en el apelativo llanga 'viga', 'madero para pasar de un lado a otro', y en algunos topónimos. Añade que «la combinación de este término con sufijo átono -ARA, probablemente prerromano, generó seguidores en nuestra toponimia [...]: Las Lláncaras, El Cuerno de Lláncara». Como puede observarse, la acentuación es proparoxítona, frente a la paroxitonía de los topónimos alaveses, a pesar de lo abundantes que son, para nuestros oídos navarros, los nombres de lugar esdrújulos en Araba. Basándonos en este criterio fonológico, parece que sería mejor dar preferencia a la hipótesis antroponímica arriba mencionada.

Markiz / Marquínez (Bernedo)

En la Reja (1025) encontramos Marquina de iuso y Marquina de suso que hacen referencia 
al actual Marquínez, villa de Bernedo, llamada en euskera Markiz, como deja de manifiesto la microtoponimia de la zona (López de Guereñu, 1989: 327). En 1087 encontramos a Gonzalvo Monnioz de Marquiniz (Ledesma, 1989, 162, p. 114), en 1257 se documenta Marquiniç (Rodríguez, 1989, 235, p. 222), que es sin duda un derivado tardío de Marquina de la Reja y la forma de la que han salido tanto Marquínez como Markiz. En 1312 tenemos todavía «Marquina de suso e Marquina de yuso» (Díaz de Durana, 1994, 14, p. 13); en 1350 hay un Johan Domingoz de Marquiniz en Villafría (Carrasco, 1973: 351).

De Marquiniz ha salido en castellano Marquinez, con final -ez habitual en los patronímicos a partir de cierta época especialmente, seguramente por acomodación al modelo fonético castellano, aunque en zonas en las que el euskera ha tenido una gran fuerza la variante en -iniz se emplea también en castellano (Gaminiz, Lauquíniz).

El nombre de base podría ser Marcius, dotado del sufijo -ina con moción de género femenino que deja claro que en la base había un *(villa) marcina 'la propiedad de Marcius'. Mitxelena ( $A V, 432)$ explica que Marquina es un derivado de marka 'marca, señal' '*frontera' pero añade que no se puede excluir la posibilidad de que tengamos un antropónimo *Marki 'Marcos', análogo a Bethiri, Pet(i)ri 'Pedro'. Ahora le podemos quitar el asterisco al *Marki de Mitxelena, pues el nombre está documentado. (véase Salaberri, 2003: 213). La forma antigua del topónimo que estamos examinando coincide con el nombre de la localidad vizcaína de Markina, pronunciada en euskera con palatal [markia], hecho que habrá impedido seguramente la caída de la nasal lene en posición intervocálica, si no se trata de una forma rehecha a partir de *Markîa (cf. Abadiño, de un anterior *Abadîo, documentado como Abadio; véase Salaberri, 2013: 258).

Otra posibilidad es pensar que el nombre de la villa vizcaína está basado en Marcinus; la evolución habría sido de ser así la siguiente: *(villa) marcinana > MarkiNâa > Markina, con tratamiento de la primera nasal como si fuere forte, reforzada por la nasalidad de la $\hat{a}$, la cual se debe a la pérdida de la segunda nasal en posición intervocálica. Otra vía sería pensar en una variante *Marcinnus, con nasal geminada que no caería. La localidad de Marquina en Zuia es Marquina en 1257 (Rodríguez, 1989, 235, p. 220), pero desconocemos cuál era la variante eusquérica de este topónimo.

\section{Okariz (Donemiliaga)}

Ocariz (1025, Reja), Senior Lope Ghideriç de Ocariç (1062, Lacarra, 1965, 22, p. 32), Ocariz (1232, Jimeno, 1970: 248; 1257, Rodríguez, 1989, 235, p. 227), Sancio Martiniz de Ocariz (c. 1275, ibíd., 340, p. 303), Ocaris (1321, Inurrieta, 1989, 40, p. 53), Ocariz (1332, ibíd., 46, p. 72 y 1351, 61, p. 96), Sancho Ruiz de Ocariz (Gereñu, 1456, Pozuelo, 1998, 6, p. 23).

En la documentación legerense, en 1066, aparece Ozchariz tres veces (Martín Duque, 1983, 78, pp. 121-122), rodeado de nombres de poblaciones alavesas y parece, por lo tanto, que se refiere a Okariz y que ha podido haber confusión entre este topónimo y el Ozkari(t)z navarro. En lo que toca a la etimología del topónimo, necesitamos para explicarlo un nombre como * Ocarius, que sin embargo no encontramos. Sí que está documentado Ocanius, que podría ser el étimo, si se acepta que, como en otras ocasiones, hay - $r$ - en lugar de la - $n$ - original en posición intervocálica (lucanica > lukarika 'longaniza'). 


\section{Petrikiz (despoblado de Arkauti-Ilarratza)}

Betriquiz (1025, Reja), Betriguiz (1135, Rodríguez, 1992, 108, p. 178), Lop Uellez de Ueitriguez, Gonzaluo Ueilez de Uetriguez (1138, ibíd., 117, p. 191), Betriquiz (1266, López de Guereñu, 1989: 648), Betriquet (1295, Rodríguez, 1989, 512, p. 439), Betriquiç (1295, ibíd., 518, p. 448), Petriquiz (1728, López de Guereñu, 1989: 648), Petriquis (s. XVIII; ibíd.).

Mitxelena $(A V, 521)$ dice lo siguiente al analizar el nombre propio Pet(i)ri que encontramos en ciertos apellidos vascos: «tal vez la var. Bet(i)ri esté representada en el nombre Betriquiz de una población alavesa de 1025». En nuestra opinión puede ser un derivado del hipocorístico Betriko, Petriko (Salaberri, 2009: 146), aunque creemos que es más acertado pensar que procede de *Betriki, con la misma base y sufijo -ki presente, por ejemplo, en Joangi, Joanki o Peruki (ibíd., 138-139), o en Andreki, base del mencionado Andrekiain (N). El final, claro está, es el sufijo -(i) (t)z.

\section{Trokoiz / Trocóniz (Iruraitz-Gauna)}

Troconiz (Reja, 1025), Trochoniz (1257, Rodríguez, 1989, 235, p. 218), Miguell Sanches de Troconis (1312, Díaz de Durana, 1994, 14, p. 13). La variante eusquérica del nombre de la localidad, que está documentada, es Trokoiz, ez decir, presenta caída de la nasal lene en posición intervocálica.

Caro Baroja (1945: 103) cree que procede de Troconici genitivo de Troconicus, fruto de añadir el sufijo -icus al antropónimo Troconius, que cita sin asterisco. Yo pienso que hay que partir de una variante no documentada *Troccus de Troccius (Solin y Salomies, 1994: 191; cf. Abundus / Abundius, Acutus / Acutius, Barbatus / Barbatius, Brutus / Brutio, Lupus / Lupius, Lupio, etc. Hay también Trocceius), seguramente declinado según el modelo -o, -onis, es decir, habrá salido de *(fundus, villa) trocconis 'la propiedad de *Troc(c)us', con la siguiente evolución: *Troconitz > Troconiz > Trokoiz.

\section{Topónimos en -ona / -o(a)}

Caro Baroja (1945: 95) empareja los finales - ana y -ona e indica que «están representados en Vizcaya por "Aramaiona", "Bedarona", "Castrejana", "Lejona”, "Lemona", "Libarona", "Luchana", "Mallona", "Quintana" y "Sopelana" por lo menos», los cuales pierden la $<\mathrm{n}>$ como los en -ano (Galdacano > Galdacao) y -iano (Ochandiano > Ochandio) y son pronunciados "Aramaio", "Lemoa", etc. Añade que en la mayoría de los casos es fácil encontrar los nombres personales que están en la base: Aramaeus, Vedius, Castricius, Lemonius, Libarius, Lucius, Mallius, Quinto.

Mitxelena $(A V, 489)$ escribe que la «terminación -ona [es] frecuente en nombres de poblaciones de Guipuzcoa y Vizcaya», que su «forma popular es -oa» y que según Caro Baroja «es fácil encontrar los personales que los compusieron». Considera el lingüista de Errenteria que dicha terminación es habitual también en apellidos, y dice, contraviniendo en parte al menos la opinión de Caro Baroja, que lo que precede a -ona en los apellidos citados (Acilona, Bertendona, Durañona, Letona, Licona...) es para él «absolutamente hermético». Concluye que no es latín ni vasco, que podría ser indoeuropeo identificable, pero que todavía no está identificado. 
Yo creo que estamos ante lo que en origen era un acusativo de nombres que en principio se declinaban por la tercera, según el modelo -o, -onis, pero que posteriormente, en latín tardío, quizás a través de su uso en acusativo (-one $(m)$ ), ha sido adaptado a la declinación -ona, -onae, por el alargamiento del tema en nasal mediante el sufijo - (vid. Irigoien, 1990: 3943, 55-60 y Villar \& Prósper, 2005: 434), como es el caso de Barcelona (< Barcin na), Pamplona (Pampilona $<$ *Pampil na), Tarragona (< Tarrac na), etc.

Viendo los topónimos de Vasconia que tienen la terminación -ona en romance y -oa (> -o en algún caso) en euskera, está claro que indican posesión, que el lugar en cuestión era en un tiempo propiedad de la persona portadora del nombre que está en la base o, más esporádicamente, lugar dedicado a la deidad que el nombre de base indica. En el segundo caso se encuentra, si no estamos equivocados, el topónimo navarro Larragoa (eusk.) / Larraona (cast.), ambos de un anterior Larrahona, construido sobre el teónimo Larrahe (o Larrahi); véase Salaberri (2007).

A continuación estudiamos los topónimos alaveses que llevan dicha terminación, pero antes queremos señalar que otros topónimos como Arroa / Arrona (G), Leioa / Lejona (B), Lemoa / Lemona (B), Zestoa / Cestona (G), etc., pueden ser explicados sin dificultad a partir de *(villa) arrona (Arrus; hace pendant con Arroi $(t) z$ y Arruitz < Arronitz en Navarra, o Arroze en Oztibarre, Baja Navarra), *(villa) leiona (Laedius, Laegius, Leius), *(villa) lebona (Laebus, con asimilación regresiva de nasalidad), *(villa) zestona (*Caestus, *Cestus, variante en -us del documentado Caestius, Cestius; el euskera habría adoptado el nombre con la velar sorda inicial asibilada en contacto con la vocal media palatal; cf. zeru, por ejemplo; la variante Çeztona está documentada).

Parece que en cierta época, como ocurre con otras terminaciones, -ona se hizo independiente y se unió a bases que, aparentemente al menos, son apelativas, aunque no se puede abandonar totalmente la idea de que se trate de nombres personales eusquéricos caídos en desuso y basados en apelativos que la lengua poseía. Este podría ser el caso, por ejemplo, de Aizkoa / Azcona (<Aizkona, quizás sobre haizko, diminutivo de haitz 'peña') o Artaxoa / Artajona (sobre *Artaxo, derivado de arte 'carrasco' y sufijo -xo que no tiene que ser por necesidad equivalente y del mismo origen que -zu en Artazu, nombre de otra localidad navarra).

A continuación pasamos a examinar los topónimos alaveses que llevan el mencionado sufijo:

\section{Abitoa / Abitona (despoblado de Agurain)}

Habitona (1257, Rodríguez, 1989, 235, p. 218), Johan de Abitona (Agurain, 1320, Iñurrieta, 1989, 32, p. 41), Martin Peres de Abitona (1320, ibíd., 36, p. 52), «... la iglesia de Habitona que es en tierra de Alava en el arçiprestazgo de Eguilaz» (1450, Goicolea, 1998: 188), «... los dichos monesterio de Uhula e lugar despoblado de Abitona» (1447, ibíd., 203), «... un lugar despoblado que se llama Abitona» (1452, Goicolea, 2002, 3, p. 4). Según López de Guereñu (1989: 612) se documenta en 1085 como Avitona y en 1086 como Abitona, que se repiten en 1719 y 1802.

La forma eusquérica estaba desprovista, como es habitual, de la nasal intervocálica:

«... el conçejo e ommes buenos de la aldea de Langarica sobre rason de un lugar que se llama Abitua e sobre los terminos e dehesas e prados e pastos del dicho lugar, el qual ha muy grand tiempo 
que fue despoblado e que el dicho lugar con los dichos prados e dehesas e pastos que es en termino e jurediçion de la dicha my villa» (Agurain, 1441, Goicolea, 1998: 164)

La forma del siglo XV muestra, igualmente, el paso -oa > -ua bien conocido en nuestra lengua. En toponimia menor hay Abito-sarria en dicho despoblado (López de Gureñu, 1989: 19), y está claro, por lo tanto, que existió una variante Abitoa, sin nasal intervocálica, que podía ser pronunciada también Abitua. En 1513 encontramos Abitoako petrala que parece significar 'Abitoako mugarria', 'el mojón de Abitona': «Mas pasaron a vna pieça del dicho Ruy/ Garcia do dizen Abitoaco Petrala, que es debaxo de la yglesia de Abitona, / al pie de vn espino, mirando el mojon azia Açilu» (Pozuelo, 2010, 84, p. 375). Este testimonio muestra claramente que la -a final, como etimológicamente le corresponde (cf., sin embargo, Aramaio), no se perdía cuando el topónimo estaba declinado.

Caro Baroja (1945: 93) ve el antropónimo Avitus en el topónimo alavés Avituana, que no sé si está por Abitona o es algo diferente. Yo creo que estamos ante un topónimo basado en el antropónimo Abitus o Avitus (Solin y Salomies, 1994: 30, 287, 299; Azkarate y García, 1996: 125, recogen el antropónimo Avit[us] en una inscripción del siglo tercero en Argote), o mejor, en una forma *Abito derivada de aquel. De * (villa) abitona 'la propiedad de *Abito' habrá salido Abitona primero y Abitôa > Abitoa después, según las reglas fonéticas del euskera. Está claro, por otra parte, que la forma Abitona no es totalmente castellana, pues tendríamos, de ser así, algo como *Abidona o *Abedona, dependiendo de la longitud de la segunda vocal.

Como en otras ocasiones, habrá que pensar que la $H$ - de los testimonios de 1257 y 1450 no es etimológica. Otra solución es considerar que la nasal del sufijo produjo una aspiración que luego, por la imposibilidad de perdurar en la cuarta sílaba, pasó a la primera: *(villa) abitona $>$ Abitona $>$ *Abitoha $>$ *Habitoa. El problema radica en que la variante documentada con $h$ - es la romance, es decir, la que no ha perdido la nasal intervocálica y no ha podido dar lugar a una aspiración. Por lo tanto, si no se piensa en una h-meramente gráfica habría que considerar que la supuesta forma vasca *Habitoa habría influido en la romance, dando lugar a Habitona, explicación bastante rebuscada sin embargo.

\section{Aramaio / Aramayona}

En uno de los romances medievales aparece nuestro topónimo usado en euskera: «... Barriz enendorque Aramaioco contrara» (Mitxelena, 1964, 3.1.6.), y de nuevo en 1448: "[Ar] amayo, suac erre açala» (ibíd., 3.1.7.). En 1467 tenemos a don Pero Martinez de Aramayona (Enríquez et al., 1994, 10, p. 70); en 1470 vivía en Arrasate Iohn Martines de Aramayo (Zumalde, 1994, 10, p. 29), que aparece en 1475 como Juan Martines de Aramayon (Crespo et al., 1996, 228, p. 412). En 1497, en Eskoriatza, encontramos a Martin de Aramayo (Lema, Cruz y Larrañaga, 2003, 19, p. 217); en la misma localidad y fecha se documenta «entre Leniz e Aramayona e Mondragon» (ibíd.).

Caro Baroja (1945: 95), como hemos visto, lo hace derivar del antropónimo Aramaeus, que nosotros no encontramos. En el caso de la localidad alavesa tendríamos que partir de *(villa) aramaiona, *(villa) alamaiona o *(villa) alabaiona, *(villa) arabaiona 'la propiedad de *Alamaeus, *Aramaeus, o de *Alabaeus, *Arabaeus' de donde Aramaiona (si el étimo tuviera una bilabial no nasal habría que pensar en una asimilación regresiva de nasalidad) > *Aramaioa > Aramaio. Arabayona de Mógica (Salamanca), llamado antes Hornillos, se debe a don Rodrigo 
de Mújica, natural de la localidad alavesa (García Sánchez, 2007: 95).

Berbijona (despoblado de Albaina, Trebiñu)

Bergilgona (1025), Verguiiona (1257, Rodríguez, 1989, 235, p. 222), «hermita de San Pedro de Berbijona» (1686, González de Viñaspre y Uribarrena, 2012: 438), S[a]n Pedro de Berbijona (1730, ibíd.).

Creo que se puede explicar partiendo de Vergilius, Virgilius, si pensamos que el grupo -rg- latino se ha mantenido en romance, como en $v$ rga > verga, al contrario de lo que parece que ha ocurrido en Vercijón (Salaberri, 2012b: 353). La evolución del topónimo es similar a la de Baroja (<*Baroxa < Varoiia, 1257 < Barolla < Barolha, 1025), es decir, habrá sido la siguiente: *(villa) Virgiliona o *(villa) Vergiliona 'la propiedad de Virgilio, Vergilio' > Bergillona (1025) > Verguiiona (1257) > *Bergixona > Berbijona (por asimilación de consonantes). En euskera esperaríamos algo como *Bergilloa, *Birgilloa, *Berbilloa, *Birbilloa.

\section{Eztarroa / Estarrona (Gasteiz)}

Este topónimo se documenta en la Reja, en 1025, como Eztarrona y en 1257 (Rodríguez, 1989, 235, p. 219) como Eçtarrona. En 1295 hay Heztarrona (ibíd., 512, p. 439), con <h->, y en 1517 y 1562 otra vez Eztarrona, que encontramos de nuevo, con la vocal inicial cambiada, en Aztarrona de 1572 (López de Guereñu, 1989: 629). Según información que me facilita E. Martínez de Madina, la variante con -zt- es mayoritaria hasta el siglo XVIII, época en la que se impone la variante con -st-. Este hecho coincide, a grandes rasgos, con el debilitamiento de la lengua vasca en la llanada alavesa, y es conocido en otras regiones de Vasconia como Navarra.

En toponimia menor, según los testimonios recogidos por López de Guereñu (1989: 206), tenemos siempre la forma con nasal: Estarronabidea, Estarronaburu(a), Estarronacidorra... pero en 1601 hay Eztarroa aldea y en 1607 Estarroarabidea. Hay, además, Estarrena- / Esterna- / Estarna- / Ezterna- en compuestos (datos de Elena Martínez de Madina), que parecen mostrar una variante Estarna-, Esterna-, Ezterna- surgida en última instancia de Eztárrona, Estárrona (o quizás de la forma asimilada *Eztérrona, *Estérrona), con pronunciación proparoxítona y caída de la vocal postónica que habría impedido la pérdida de la nasal, al no estar ya en posición intervocálica. Parece, como la mayoría de los topónimos en -ona (cast.) /-oa (eusk.), un nombre de lugar con base antroponímica, que tendría que ser algo como *(E)starrus, *(E)starro, un nombre indígena no identificado, pero nos movemos aquí en el terreno de las hipótesis.

Iroxona (despoblado de la zona de Egino y Agurain)

Irossona (Reja, 1025). Si el sufijo es -ona como parece, la variante vasca habrá sido algo como *Iroxoa y la base será *Irox(o)-, tal vez de carácter antroponímico.

Ixona / Hijona (Burgu / Elburgo)

Se documenta como Iscona (Reja, 1025), Exona (1257, Rodríguez, 1989, 235, p. 218), 
don Martin de Asona (c. 1275, ibíd., 340, p. 304), Ajona (1294), Axona (1294, s. XVI), Axona (1295, ibíd., 512, p. 438), Ixona (1593), Yjona (1694), Ijona (s. XIX) (López de Guereñu, 1989: 633), pero no sabemos qué variante se utilizaba en euskera, ya que no disponemos de ningún testimonio de su uso en microtoponimia.

La Comisión de Onomástica de Euskaltzaindia ha normativizado el nombre como Ixona, pero da la impresión de que faltan datos de toponimia menor que corraboren o refuten dicha forma. Nosotros creemos que es posible que se usara *Ixoa o * Exoa, es decir, la variante sin la nasal intervocálica, lo que nos llevaría a un étimo *(villa) isiona, con palatalización de la sibilante en contacto con la vocal o vocales palatales, pero el único antropónimo que encuentro es Esius que Solin y Salomies (1994: 75) recogen con un interrogante. Sería posible, igualmente, basarse en algo como *Exius [eksius], *Ixius [iksius], es decir, partir de *(villa) exiona, ixiona, pero no hay nada así, que sepamos.

También podría ser considerado un topónimo con sufijo -ana $>-a$, si partiéramos de una variante *Hisonus de Hisonius que Solin y Salomies (ibíd., 93) dan con interrogante. En este caso la evolución habría sido: *(villa) isonana > isonâa (con pérdida de la nasal lene en posición intervocálica, que antes ha nasalizado la vocal precedente; esta nasalización habrá reforzado la nasalidad de la primera $n$ que ha sido tratada como si fuera $N$ ) $>{ }^{*}$ IsoNa $>$ Ixona (con palatalización IsV- > IxV-).

\section{Letona (Zigoitia)}

En el año 871 se documenta (Ubieto, 1976, 10, p. 20) Letonu junto a Zeztave, entre otros, por lo que, como quería López de Guereñu (1989: 638), podemos estar ante un variante de Letona (hay también Ganna por Gauna, al parecer) que se documenta por primera vez así, tal cual, en 1093 (López de Guereñu, 1989: 638) y se repite en 1173 (Rodríguez, 1979, 254, p. 30), 1257 (Rodríguez, 1989, 235, p. 219) y 1295 (ibíd., 512, 519, pp. 438 y 447).

En toponimia menor López de Guereñu (ibíd., 302) trae Letonabide y Letonacorta, formas en las que parece recogerse la variante oficial del nombre, no la eusquérica que sería *Letoa, a no ser que partiéramos de un antropónimo *Laeton(n)us. Irigoien (1986: 42) cree que deriva del antropónimo Leto que aparece (Leto testis) en la documentación de San Millán de la Cogolla.

Considero que la propuesta de Irigoien es acertada; Leto procederá del documentado Laetus, y el topónimo habrá salido de dicho antropónimo declinado según el modelo -o, -onis (Leto, Letonis), de cuyo caso oblicuo alargado (vid. Aramaio / Aramayona) procederá Letona, a partir de *(villa) letona 'propiedad de Laetus' y *Letoa después, con perdida de la nasal lene en posición intervocalica. Puede pensarse, igualmente, que la forma Letona- documentada era la que se empleaba en euskera; en este caso habría que partir de la supuesta variante *Laeton(n) us provista del sufijo -ana: *(villa) leton(n)ana $>$ *LetoNâa $>$ Letona.

\section{Marantxona (despoblado de Etura-Gebara, Barrundia)}

Maranchona (1257, Rodríguez, 1989, 235, p. 218), Maranchona (1295, ibíd., 512, p. 438), Marranchona (1715, López de Guereñu, 1989: 640). Este mismo autor recoge Maranchonalavista en 1716 como labrantío de Audika / Audicana, que parece una tradución castellana de una forma vasca con -agerri, del tipo de Iruñagerria en Kordoba / Cordobilla, 
topónimo que designa el lugar desde el que se ve Pamplona en este caso, o Gendulainagerria de Galar, equivalente al castellano Cara (a) Gendulain (Jimeno, 2011: 129, 242, 249). En 1718 hay Maranchon «arroyo de Audicana» (López de Guereñu, 1989: 323). La forma eusquérica que esperábamos pero que no está documentada, que nosotros sepamos, es *Marantxo(a).

Creo que se podría partir de *(villa) valentiona, a partir del antropónimo Valentius o Valentio, si se aceptara que ha habido palatalización - tjV- > - txV- que se da en otros topónimos alaveses como Aletxa, Andetxa, Gometxa y *Lopetxa (véase Salaberri, 2012), y que se ha producido el paso $b$ - > m-, favorecido por la(s) nasal(es) del topónimo. La evolución -l->-r- es habitual en posición intervocálica, y una asimilación $a-e>a-a$ creo que no tiene nada de extraño.

\section{Ondoa / Ondona (Goiuri-Ondona, Urkabustaiz)}

Ondona (937, López de Guereñu, 1989: 645), Undona (1257, Rodríguez, 1989, 235, p. 229), Undona (1770, López de Guereñu, 1989: 645; cita de Landázuri). Parece tratarse de un derivado del nombre no documentado *Ondo, *Onto; es decir el topónimo podría proceder de un étimo como *(villa) ontona, * (villa) ondona. El paso $o>$ u en contacto con nasal es conocido (cf. Mungia, B, derivado del antropónimo Boncius). En euskera se empleaba la forma Undua, sin duda de una anterior *Undoa (véase lo dicho en Abitoa / Abitona), como demuestran los microtopónimos Unduagan (1807, alto de Izarra-Abezia), Unduarbin (1761, término de Izarra) (López de Guereñu, 1989: 478).

\section{Bibliografía}

Arana, S., 1980 (1897), Tratado Etimológico de los apellidos euskéricos, in Obras completas de Arana Goiri'ttarr Sabin, vol. I, segunda edición, Donostia / San Sebastián, 702-809.

Ayerbe, M. R., 2006, Documentación Medieval del Archivo Municipal de Segura. Tomo III (14501521), Fuentes documentales medievales del País Vasco 127, Eusko Ikaskuntza, Donostia / San Sebastián.

Azkarate, A. y García, I., 1996, Estelas e Inscripciones Medievales del País Vasco (Siglos VIXI). I. País Vasco Occidental / Euskal Herriko Erdi Aroko Hilarri eta Inskripzioak (VI-XI. mendeak). I. Euskal Herriko Mendebaldea, Universidad del País Vasco / Euskal Herriko Unibertsitatea - Gobierno Vasco, Bilbo / Bilbao.

Caro Baroja, J., 1945, Materiales para una historia de la lengua vasca en su relación con la latina, Salamanca. Existe una edición más reciente realizada por Txertoa en Donostia / San Sebastián en 1990.

, 1980, Historia General del País Vasco, vol. III, La Gran Enciclopedia Vasca, Luis Haramburu ed., Bilbo / Bilbao.

Carrasco, J., 1973, La Población de Navarra en el siglo XIV, Universidad de Navarra, Iruñea / Pamplona.

Crespo, M. A. et al., 1995, Colección Documental del Archivo Municipal de Bergara. Tomo I (1181-1487), Fuentes documentales medievales del País Vasco 57, Eusko Ikaskuntza, Donostia / San Sebastián.

, 1996, Colección Documental del Archivo Municipal de Mondragón. Tomo IV. (1471-1500), Fuentes documentales medievales del País Vasco 69, Eusko Ikaskuntza, Donostia. 
Del Valle Lersundi, F., 1933, "Una forma del femenino y el valor de la letra «ch» como diminutivo en los nombres de los guipuzcoanos de los siglos XV y XVI", RIEV 24, 176-181.

Díaz de Durana, J. R., 1994, Alava en la Baja Edad Media a través de sus textos, Fuentes documentales medievales del País Vasco 54, Eusko Ikaskuntza, Donostia / San Sebastián.

Enríquez, J. et al., 1994, Colección Documental del Archivo Municipal de Orduña (1271-1510). Tomo I, Fuentes documentales medievales del País Vasco 52, Eusko Ikaskuntza, Donostia / San Sebastián.

Garate, J., 1931, "Sufijos locativos", RIEV 21, 442-448.

García Arias, X. Ll., 2005, Toponimia asturiana. El porqué de los nombres de nuestros pueblos, La Nueva España, Oviedo.

García Larragueta, S., 1957, El Gran Priorado de la Orden de San Juan de Jerusalén. Siglos XIIXIII. Colección Diplomática, Institución Príncipe de Viana, Iruñea / Pamplona.

García Sánchez, J. J., 2007, Atlas toponímico de España, Arco/Libros, Madrid.

Goicolea, Fco. J., 1998, Archivo Municipal de Salvatierra-Agurain. Tomo II. (1401-1450), Fuentes documentales medievales del País Vasco 83, Eusko Ikaskuntza, Donostia / San Sebastián.

,2002, Archivo Municipal de Salvatierra-Agurain. Tomo III. (1451-1500), Fuentes documentales medievales del País Vasco 115, Eusko Ikaskuntza, Donostia / San Sebastián.

Gómez-Moreno, M., 1925, "Sobre los íberos y su lengua", Homenaje a Menéndez Pidal, vol. III, Madrid, 475-499.

González de Viñaspre, R. y Uribarrena, P., 2012, "Los despoblados medievales del Condado de Treviño / Trebiñuko Konderriko Erdi Aroko herri hustuak", Viaje a Íbita. Estudios históricos del Condado de Trevino, Ayuntamiento del Condado de Treviño, VitoriaGasteiz, 427-450.

Goñi, J., 1997, Colección Diplomática de la Catedral de Pamplona. 829-1243, Gobierno de Navarra, Iruñea / Pamplona.

Iglesias, H., 2001, "Aztarna germanikoa Euskal Herriko toponimia historikoan”, FLV 87, 317 335.

, 2002, "Sur le toponyme Gasteiz: origine et signification", FLV 89, 109-138.

Iñurrieta, E., 1983, Cartulario Real a la Provincia de Álava (1258-1500), Fuentes documentales medievales del País Vasco 3, Eusko Ikaskuntza, Donostia / San Sebastián.

, 1989, Colección Diplomática del Archivo Municipal de Salvatierra (1256-1400), Fuentes documentales medievales del País Vasco 18, Eusko Ikaskuntza, Donostia / San Sebastián.

Irigoien, A., 1982, "Sobre el topónimo Gasteiz y su entorno antroponímico", Vitoria en la Edad Media, Actas del I Congreso de Estudio Históricos celebrado en esta ciudad del 21 al 26 de setiembre de 1981, en conmemoración del 800 aniversario de su fundación, Ayuntamiento de Vitoria-Gasteiz, Vitoria-Gasteiz, 621-652.

, 1986, En Torno a la toponimia vasca y circumpirenaica, Deustuko Unibertsitatea, Bilbo / Bilbao.

, 1987, "Sobre el origen de los patronímicos y de ciertos topónimos terminados en -ain, -ein, sul. -añe", in De Re Philologica Linguae Uasconicae II, Bilbo / Bilbao, 193-207.

, 1990, Sobre Toponimia del País Vasco Norpirenaico (Observaciones en torno a la obra Toponymie 
Basque de Jean-Baptiste Orpustan), Universidad de Deusto, Bilbo / Bilbao.

Jimeno, J. Mª, 1968, Documentos Medievales Artajoneses (1070-1312), Diputación Foral de Navarra, Institución Príncipe de Viana, Iruñea / Pamplona.

, 1970, "El libro rubro de Iranzu", PV 120-121, 221-269.

, 2011, Toponimia navarra IV. Cuenca de Pamplona. Cendea de Galar, Ayuntamiento de Galar Udalbide - Pamiela - Euskara Elkargoa, Obras completas de José M ${ }^{a}$ Jimeno Jurío, ${ }^{\circ}$ 43, Iruñea / Pamplona.

Lacarra, J. M., 1965, Colección Diplomática de Irache. Volumen I (958-1222), CSIC, Zaragoza.

Lafon, R., 1980 (1944), Le système du verbe basque au XVI siècle, Elkar, Donostia / San Sebastián.

Ledesma, Mª. L., 1989, Cartulario de San Millán de la Cogolla (1076-1200), Instituto de Estudios Riojanos - Monasterio de San Millán de la Cogolla - Anúbar ediciones, Zaragoza.

Lema, J. A., 2007, Colección Documental del Archivo Municipal de Bergara. II. Fondo Municipal: Subfondo Histórico (1335-1520), Fuentes documentales medievales del País Vasco 133, Eusko Ikaskuntza, Donostia / San Sebastián.

, Cruz, J. R. y Larrañaga, M., 2003, Archivos municipales (1260-1520): Antzuola (1489-1497), Aretxabaleta (1506), Eskoriatza (1260-1519) y Leintz-Gatzaga (Salinas de Léniz) (13721516), Fuentes documentales medievales del País Vasco 116, Eusko Ikaskuntza, Donostia / San Sebastián.

López de Guereñu, G., 1989, Toponimia alavesa seguido de Mortuorios y despoblados y Pueblos alaveses, Onomasticon Vasconiae 5, Euskaltzaindia, Bilbo / Bilbao.

Martín Duque, A., 1983, Documentación Medieval de Leire (siglos IX a XII), Diputación Foral de Navarra, Príncipe de Viana, Iruñea / Pamplona.

Martínez de Madina, E., 2010, Toponimia de Vitoria III / Gasteizko Toponimia III, Onomasticon Vasconiae 29, Euskaltzaindia, Bilbo / Bilbao.

Mitxelena, K., 1950, "Notas etimológicas vascas", Emerita 18, 467-481.

, 1956, "Introducción fonética a la onomástica vasca", Emerita 24, 167-186 y 331-352.

, 1957, "El genitivo en la onomástica medieval", Emerita 25, 134-148.

, 1964, Textos arcaicos vascos, Minotauro, Madrid.

, 1972, "Nota marginal sobre la huella latina en la lengua vasca", FLV 10, 2-25.

, 1997, Apellidos Vascos (AV), quinta edición, Txertoa, Donostia / San Sebastián.

Pozuelo, F., 1998, Documentación de la Cuadrilla de Campezo: Arraia Maeztu, Bernedo, Campezo, Lagran y Valle de Arana (1256-1515), Fuentes documentales medievales del País Vasco 88, Eusko Ikaskuntza, Donostia / San Sebastián.

, 2008, Archivo Municipal de Salinas de Añana - Gesaltza. Documentos (1400-1517), Fuentes documentales medievales del País Vasco 131, Eusko Ikaskuntza, Donostia / San Sebastián.

,2010, Archivo Municipal de Salvatierra - Agurain. Tomo IV (1501-1521). Apéndice 1259-1469, Fuentes documentales medievales del País Vasco 141, Eusko Ikaskuntza, Donostia / San Sebastián.

Rodríguez, I., 1979, Colección Diplomática medieval de la Rioja. Tomo III: Documentos (11681225), Servicio de Cultura de la Diputación de la Rioja, Logroño.

, 1989, Colección Diplomática medieval de la Rioja. Tomo IV: Documentos siglo XIII, Gobierno de La Rioja, Instituto de Estudios Riojanos, Logroño.

, 1992 (1976), Colección Diplomática medieval de la Rioja. Tomo II: Documentos (923-1168), 
Gobierno de La Rioja, Instituto de Estudios Riojanos, Logroño.

Ruiz de Lóizaga, S., 1997, Documentación Medieval de la Diócesis de Vitoria en el Archivo Vaticano (Siglos XIV-XV), Zaragoza.

Salaberri, P., 2003, Euskal Deiturategia: Patronimia, Udako Euskal Unibertsitatea, Bilbo / Bilbao.

, 2005, "Los trabajos de onomástica en Fontes Linguae Vasconum”, FLV 100, 607-632.

, 2007, "En torno a la forma vasca del topónimo mayor Larraona (Ameskoa)", Euskera 52, 395-397.

, 2009, Izen ttipiak euskaraz, Onomasticon Vasconiae 26, Euskaltzaindia, Bilbo / Bilbao.

, 2011, "Sobre el sufijo occidental -ika y otras cuestiones de toponimia vasca", FLV 113, 139176.

, 2011b, "De toponimia vasco-pirenaica: sobre el sufijo -otz, -oz(e)", Nouvelle Revue d'Onomastique 53, 33-63.

, 2011c, "País Vasco y Navarra", Toponimia Hispánica. Origen y evolución de nuestros topónimos más importantes, X. Ll. García Arias coord., E. Casanova ed., Denes, Valencia, 63-97.

, 2012, "Notas de toponimia alavesa: antropotopónimos en -a/-ana", Viaje a Íbita. Estudios históricos del Condado de Trevino, R. González de Viñaspre y R. Garay (eds.), Ayuntamiento del Condado de Treviño, Trebiñu / Treviño, 209-228.

, 2012b, "Topónimos alaveses de base antroponímica terminados en -ain, -egi, -eta (-keta), -ika, -iku (-iko, -ika), -inu (-ina), -itu (-ita), -ón", FLV 115, 323-357.

, 2013, "Topónimos alaveses de base antroponímica acabados en -(i)ano", FLV 116, 245-271.

Solin, H. y Salomies, O., 1994, Repertorium nominum gentilium et cognominum latinorum, segunda edición, Olms - Weidmann, Hildesheim - Zürich - New York.

Ubieto, A., 1976, Cartulario de San Millán de la Cogolla (759-1076), Instituto de Estudios Riojanos - Monasterio de San Millán de la Cogolla - Anúbar Ediciones, Valencia.

Villar, F. y Prósper, B. M., 2005, Vascos, celtas e indoeuropeos. Genes y lenguas, Universidad de Salamanca, Salamanca.

Zumalde, I, 1994, Colección Documental del Archivo Municipal de Oñati (1149-1492), Fuentes documentales medievales del País Vasco 50, Eusko Ikaskuntza, Donostia / San Sebastián. 
